\title{
Reviewers, 1994
}

Harold Adams

Jack Antel

H.J.M. Barnett

Paul Bedard

Brien Benoit

Timothy J. Benstead

Mark Bernstein

Henry Berry

Gilles Bertrand

John Bilbao

Warren Blume

Donald Borrett

Jean-Pierre Bouchard

Garth M. Bray

Vera Bril

Michael H. Brooke

John Brown

Gregory Cairncross

Donald Calne

Peter Camfield

Louis R. Caplan

Peter Carlen

Sterling Carpenter

Colin Chalk

Doug Cochrane

Bernard Corenblum

Robert Cote

R. Del Maestro

Allan Detsky

James Drake

Robert Duke

Pierre Duquette

Peter Dyck

George Ebers

Andrew Eisen

Kost V. Elisevich

David Fairholm

Thomas Feasby

Michael Fehlings

Gary Ferguson

J. Max Findlay

William Fletcher
Gordon Francis

Morris Freedman

Dorcas Fulton

Serge Gauthier

M. John Gill

John Girvin

Trevor Gray

Gregory Gruener

Alan Guberman

Anthony Hakim

Mark Hallett

William C. Halliday

Robert R. Hansebout

Julian Hoff

Herman Hugenholtz

Peter Humphreys

Michael Hunter

Paul Hwang

Robert Inman

Joe Jankovic

Jack H. Jhamandas

Harry J. Karlinsky

George Karpati

Andrew Kertesz

Andrew Kirk

Anthony E. Lang

Richard Leblanc

Mary Anne Lee

Donald H. Lee

Robert G. Lee

William J. Logan

Vernon H. MacMillan

Mark Malkin

C.E. Maxner

David A. McCrea

Hewby McFarland

Edith McGeer

Dan McIntyre

Richard S. McLachlan

David McNeely

David Mikulis

David G. Munoz
Theodore L. Munsat

John T. Murphy

S. Terence Myles

S. Nag

John W. Norris

Charles Olanow

Sidney J. Peerless

Richard W. Price

William Pryse-Phillips

Sherrill Purves

A.H. Rajput

N. Barry Rewcastle

Richard Riopelle

James Rutka

Dessa Sadovnick

Harvey B. Sarnat

Brian Schmidt

Michael L. Schwartz

S. Seshia

Paul Shelton

A.L. Sherwin

Ashfaq Shuaib

Anders A. Sima

Richard Stein

Paul Steinbok

John D. Stewart

A. Jon Stoess

Peter Stys

Garnette Sutherland

James A. Taren

William G. Tatton

Margot Taylor

Hillar Vellend

Jean-Guy Villemure

Michael Wall

Chris Wallace

Brian Weinshenker

Bryce Weir

John Wherrett

Uwe Windhorst

G. Bryan Young

Douglas Zochodne 